\title{
Effects of early intravenous low-dose of metoprolol on cardiac sympathetic activities and electrophysiological properties in myocardial infarction heart
}

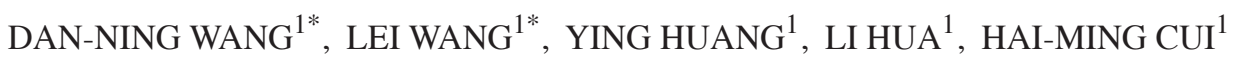 \\ PENG-FEI CHEN $^{2}$, XIN LIANG $^{1}$, JIA-YOU ZHANG ${ }^{1}$ and DE-NING LIAO ${ }^{1}$ \\ ${ }^{1}$ Department of Cardiology, Shanghai Changzheng Hospital, Shanghai 200433; ${ }^{2}$ Department of Cardiology, \\ The 309th Hospital of Chinese People's Liberation Army, Beijing 100193, P.R. China
}

Received February 12, 2018; Accepted August 6, 2018

DOI: $10.3892 /$ etm.2018.6695

\begin{abstract}
This study observed the effects of early intravenous low-doses of metoprolol on cardiac sympathetic activities and electrophysiological properties in myocardial infarction (MI) dogs. Thirty two mongrel dogs with the first diagonal branch of the left anterior descending coronary artery ligated were randomly divided into three groups: The low-dose group was given metoprolol $0.6 \mathrm{mg} / \mathrm{kg}$ immediately by intravenous injection $(\mathrm{n}=12)$; the target-dose group was given metoprolol $1.6 \mathrm{mg} / \mathrm{kg}(\mathrm{n}=12)$, and the control group was injected with normal saline at the same dose of the target-dose group $(n=8)$. Norepinephrine (NE) and epinephrine (E) levels in the coronary sinus (CS) blood as well as the ventricular effective refractory period (ERP) were all measured during the experiments. We found that $\mathrm{NE}$ and $\mathrm{E}$ concentrations in the three groups were all increased compared with the previous measurement before ligation. ERP values after MI were significantly decreased in all three groups compared with the first measurements. The three groups all exhibited uneven shortness of ERP among different regions, with significant shortness in infarcted area. Furthermore, there was no difference between the low and target-dose of metoprolol in the reduction of regional ERP, and the same effect was also observed in induced arrhythmias. In conclusion, a lower dose of metoprolol performed similarly as target-dose in reducing the catecholamine concentrations in dogs with MI. Our study demonstrated that a lower dose of metoprolol may be reasonable compared with the target-dose in $\beta$-blocker therapy due to similar effect and lower toxicity.
\end{abstract}

Correspondence to: Dr De-Ning Liao, Department of Cardiology, Shanghai Changzheng Hospital, 415 Fengyang Road, Shanghai 200433, P.R. China

E-mail:wdn861921310@163.com

${ }^{*}$ Contributed equally

Key words: metoprolol, $\beta$-blocker, myocardial infarction, effective refractory period

\section{Introduction}

Coronary heart disease (CAD) is the most common type of organ disease caused by atherosclerosis and a common disease that is harmful to human health. With the improvement of people's living standards and the arrival of an aging society, the incidence of CAD increases year by year, and gradually becomes the first cause of death $(1,2)$. Studies worldwide have shown that the incidence of premature CAD is increasing, and the course of disease is developing rapidly. The condition is dangerous, and the rate of sudden death is high (3). The patients with CAD usually have no symptoms, and the clinical manifestations are mainly myocardial infarction (MI). Patients with CAD, especially when they have had MI, have more cardiovascular risk factors, and comprehensive intensive interventions, including treatment of lifestyle changes, are needed to reduce the risk of future CAD and cardiovascular events $(4,5)$.

CAD or MI patients often have a family history (6). It is generally believed that CAD is characterized in the context of genetic susceptibility factors that are exposed to an atherosclerotic environment throughout lifetime. In this context, the rapid evolution of large-scale human research and genetic technology over the past few years has revolutionized our understanding of the genetic basis of CAD (7). In addition, coronary morphological information may be an important additional feature of disease prediction in patients with CAD or MI (8). The study suggests that vascular risk factors explain only $90 \%$ of the risk of MI, suggesting that the significance of genetic risk is low (9). However, this ignores the risk factors such as hypertension, hypercholesterolemia, diabetes, and even addictive behaviors (smoking), which are seriously affected by genetic factors $(10,11)$. Patients with CAD are always accompanied by impairment of cardiac and endothelial function. Combined with a previous study that risk factors, such as hypertension, obesity, smoking and abnormal metabolism of serum lipids, can mediate vascular endothelial injury by inflammation and oxidative stress (12), resulting in the change of structure and function of endothelial cells, which is considered to be the initiating factor of atherosclerosis (13). Thus, variability in performance for MI and CAD cannot be explained solely by 
frequency changes in vascular risk factors $(14,15)$, suggesting that racial or genetic differences have important clinical implications in disease pathogenesis.

Since 1980 s, $\beta$-blockers have been used in patients with CAD, especially when they have had MI (16). It is known that $\beta$-blockers improve survival following MI. This has been established in multiple randomized trials including CAPRICORN (17). $\beta$-blockers can slow down heart rate, lower blood pressure, suppress the sympathetic nerve system, and improve myocardial oxygen supply and demand imbalance, so as to reduce the incidence of fatal arrhythmias after MI. For this reason, the 1990 American College of Cardiology/American Heart Association (ACC/AHA) guidelines (18) first recommended (class I or II a recommendation) $\beta$-blocker therapy for essentially all post-MI patients, except those with contraindications.

Although several studies have documented under dosing of $\beta$-blockers, these studies showed that the majority of patients delivered with $\beta$-blockers did not achieve the target-doses demonstrated to be effective in the randomized trials $(19,20)$. In fact, most of the patients received $<50 \%$ of the target-dose. The randomized clinical trials did not assess the effects of different doses of $\beta$-blockers. Furthermore, there have been no large-scale studies that have addressed this topic. Thus, a hypothesis was made that a lower dose of $\beta$-blockers may present a similar curative effect with lower toxicity as the target-dose which has been demonstrated in previous studies.

Our study evaluated the effects of different doses of $\beta$-blockers on ventricular electrophysiological characteristics and induced ventricular arrhythmias in canines after MI to explore whether the lower doses may result in equivalent outcomes compared with the target-dose. These data support the need for further testing to determine optimal dosing of $\beta$-blockers after MI.

\section{Materials and methods}

Experimental models. Thirty-two mongrel dogs (16 males and 16 females) were randomly divided into the control group $(n=8)$, the low-dose group $(n=12)$ and the target-dose group $(n=12)$. The dogs were anesthetized with sodium pentobarbital (30 mg/kg, i.v.), intubated, and ventilated with room air supplemented with oxygen from a respirator (LTV-1000, Pulmonic Systems, USA). Additional maintenance doses of $2 \mathrm{mg} / \mathrm{kg}$ sodium pentobarbital were administered at the end of each hour during the procedure. Standard surface electrocardiogram (ECG) was continuously monitored by using a computer-based Lab System (TOP 2001; Hongtong Biology Technology Company, Shanghai, China). Animal handling was performed in accordance with the Shanghai Directive for Animal Research, and the present Guidelines for the Care and Use of Laboratory Animals published by the National Institutes of Health (NIH publication no. 85-23, revised 1996). The Ethics Committee of the Second Military Medical University (Shanghai, China) approved the study protocol.

Acute ischemia protocol. After anesthetized, the first diagonal artery was isolated in all groups, and then occluded by ligature (3-0 silk) for $1 \mathrm{~h}$ until the ischemic part turned dark red so as to make sure that the ligation was successful. ECG was recorded and analyzed continuously before and after MI. To achieve a stable status, we gave the animals a 90 min-pause to make sure the ECG would not change any further before proceeding.

Treatment. After ligation, the low-dose group was given metoprolol at the dose of $0.6 \mathrm{mg} / \mathrm{kg}$ immediately by intravenous injection, the target-dose group was given $1.6 \mathrm{mg} / \mathrm{kg}$, while the control group was injected with normal saline at the same dose as the target-dose group.

Measurement of plasma norepinephrine (NE) and epinephrine $(E)$. An hour after the ligation, blood samples were drawn into heparinized tubes through a modified Morawitz cannula, which was introduced into the coronary sinus (CS) through the azygos vein. All samples were placed immediately on ice after collection and centrifuged at $4^{\circ} \mathrm{C}$ within $30 \mathrm{~min}$. Plasma was collected and stored at $-20^{\circ} \mathrm{C}$ for further analysis. $\mathrm{NE}$ and $\mathrm{E}$ levels in plasma were measured by high-performance liquid chromatography (HPLC).

Electrophysiological measurements. Two multi-electrode catheters with $1 \mathrm{~cm}$ inter electrode distance were sutured to evaluate effective refractory periods (ERP) at six epicardial sites from the apex to the base of the left ventricular free walls. The ventricular ERP in each site was determined by programmed pacing that consisted of eight drive stimuli (S1) followed by an extra-stimulus (S2) at twice threshold pacing current with a $2 \mathrm{~ms}$ pulse duration. The interval between S1 and S2 was progressively decreased until refractoriness was achieved. The ERP was defined as the longest S1S2 interval that failed to capture the ventricles as described previously. ERPs were measured at the baseline and an hour after ligation. ERP dispersion was defined as the coefficient of variation (CV) of the ERP at all six sites.

Measurement of ventricular arrhythmia occurrence. Electrocardiogram was continuously monitored for $1 \mathrm{~h}$ to record the occurrence and duration of ventricular arrhythmias including ventricular premature contraction (VPC, identifiable premature QRS complexes), ventricular tachycardia (VT, three or more consecutive VPCs at a rate faster than the resting sinus rate) and ventricular fibrillation (VF, unidentifiable and low voltage QRS complexes). Especially, if VT progresses within a few beats to VF (there are no sinus beats between VT and VF), we classified these VTs as VF.

Statistical analysis. Data are expressed as mean \pm SD. NE and E changes were tested by the independent-sample t-test. ERP changes were tested by the paired t-test. Pairwise comparisons between different regions were calculated by the LSD method. Data were analyzed by using SPSS21.0 software (IBM Corp., Armonk, NY, USA). $\mathrm{P}<0.05$ was considered to indicate a statistically significant difference.

\section{Results}

Metoprolol decreases the NE and E levels in CS blood after $M I$. Since NE and E is a major cardiac stimulator and contribu tes to ventricular arrhythmias induction, we investigated whether low and target-dose of $\beta$-blocker, metoprolol, affects 
A

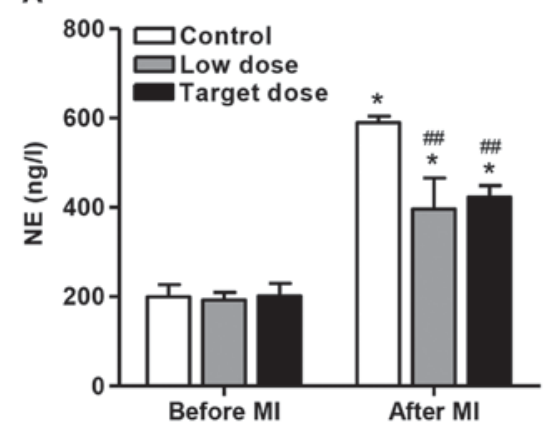

B

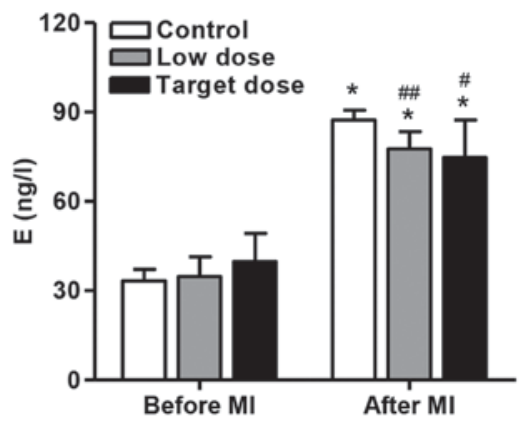

Figure 1. NE and E levels in CS blood before and after MI. (A) NE level in CS blood before and after MI. (B) E level in CS blood before and after MI. *p<0.01 compared with the groups before MI. ${ }^{\#} \mathrm{p}<0.05,{ }^{\# \#} \mathrm{p}<0.01$ compared with the control group after MI. NE, norepinephrine; E, epinephrine; CS, coronary sinus; MI, myocardial infarction.
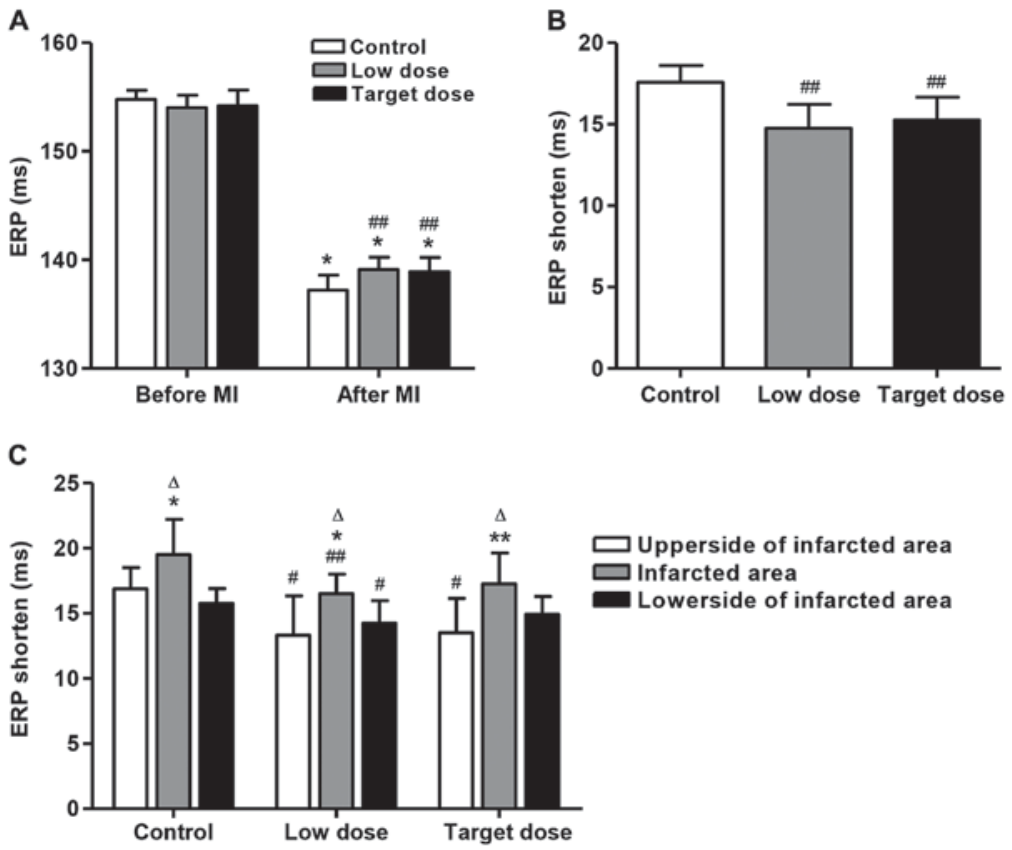

Figure 2. Changes of ERP at 6 epicardial sites in left ventricular before and after MI. (A) ERP changes at 6 epicardial sites before and after MI. "p<0.01 compared with the three groups before MI. ${ }^{\# \#} \mathrm{p}<0.01$ compared with the control group after MI. (B) ERP shortening in each group after MI. ${ }^{\# \#} \mathrm{p}<0.01$ compared with the control group. (C) ERP shortening in different areas after MI. ${ }^{*} \mathrm{p}<0.05,{ }^{* *} \mathrm{p}<0.01$ compared with the upperside of infracted area in the three groups. ${ }^{\Delta} \mathrm{p}<0.01$ compared with the lowerside of infracted area in the three groups. ${ }^{\# p}<0.05,{ }^{\# \#} \mathrm{p}<0.01$ compared with the control group in the low- and target dose groups. ERP, effective refractory period; MI, myocardial infarction.

the NE and E content differently in blood. As expected, NE and $\mathrm{E}$ levels in CS blood dramatically increased in all three groups after MI compared with that before MI (Fig. 1A and B). Moreover, the NE and E levels in CS blood were significantly decreased in both the low- and target-dose groups compared with the control group after MI $(\mathrm{p}<0.01)$, but no difference was found among the three groups in the NE and $\mathrm{E}$ levels before MI. The NE and E levels in CS blood with the low-dose of metoprolol treatment after MI was decreased by 32.7 and $11.1 \%$ compared with the control group, respectively $(\mathrm{p}<0.01)$. The NE and E levels in CS blood with the target-dose of metoprolol treatment after MI was decreased by 28.3 and $14.5 \%$ compared with the control group, respectively $(\mathrm{p}<0.01)$. However, there was no difference between the lowand target-dose of metoprolol treatment in the levels of NE and $\mathrm{E}$ in CS blood after MI. These results suggest that metoprolol decreases the NE and E levels in CS blood after MI in a doseindependent manner.

Metoprolol inhibits ventricular ERP shortening after MI. Fig. 2A summarizes the ventricular ERP changes at 6 epicardial sites in all groups before and after MI. As a result, ventricular ERP was significantly decreased in all three groups after MI compared with that before MI $(\mathrm{p}<0.01)$, but no difference was found among the three groups in ventricular ERP before MI. After MI, low- and target-dose of metoprolol increased the ventricular ERP by 1.38 and $1.24 \%$ compared with the control group, respectively $(\mathrm{p}<0.01)$. However, ventricular ERP in the low-dose group did not change significantly compared with that in the target-dose group. Moreover, after MI, low- and target-dose of metoprolol decreased the ventricular ERP shortening by 16.1 and $13.1 \%$ compared with the control group, 
respectively (Fig. 2B; p<0.01). However, ventricular ERP in the low-dose group did not change significantly compared with that in the target-dose group.

In addition, to make sure whether there was any difference in ERP prolongation among the sites which were above, in and below the infracted area, we calculated and compared the average ERPs of the two sites above the infracted area (upper sides), two sites in the area (infarcted area) and two sites below the area (lower sides). Then, we calculated the diffe rence between these 3 areas. We found that ERP shorten in the infarcted area was significantly increased compared to those in the other two areas in all three groups (Fig. $2 \mathrm{C}$; $\mathrm{p}<0.05$ ). However, no significant difference between the upper and lower sides of the infarcted area was detected. The same trend was found in the low- and target-dose groups. Moreover, the low and target-dose of metoprolol significantly decreased the ERP shorten by 21.0 and $19.9 \%$ in the upper side of infracted area compared with the control groups, respectively (Fig. $2 \mathrm{C}$; $\mathrm{p}<0.05)$. However, only the low-dose of metoprolol treatment could decrease the ERP shortening by 15.4 and $9.5 \%$ in the infracted area and in the lower side of the infracted area compared with the control groups, respectively $(p<0.05)$. These results indicate that the low-dose of metoprolol treatment ERP shortening was found above, in and below the infracted area.

Effect of low- and target-dose of metoprolol on ventricular arrhythmia occurrence. The episodes of PVCs and VT as well as the mean duration of VT in the low-dose group have no significant changes when compared to the high-dose group. 5 of $12(41.7 \%)$ animals in the low-dose group had spontaneous VF compared to 4 of $12(33.3 \%)$ animals in the target-dose group $(\mathrm{p}>0.05)$.

\section{Discussion}

In this study, we investigated the effects of early intravenous low-doses of metoprolol on ventricular electrophysiological properties by using an acute MI canine model. The results indicated that the low-dose of metoprolol exhibited the same effects on the level of NE and E as well as the ERP in animals with MI. These results suggest that the low-dose of $\beta$-blockers may exert a similar protective effect against ventricular arrhythmias after MI compared with the target-dose of $\beta$-blockers.

At present, the common measures we take for the prevention of arrhythmias after MI include $\beta$-blocker therapy, implanta ble cardioverter-defibrillator (ICD) and other antiarrhythmic medications. $\beta$-blockers have been proven to be effective in slowing heart rate, decreasing myocardial contractility and lowering blood pressure. Since the prevalence of $\beta$-blockers in patients after MI, $\beta$-blockers were used at a lower-dose than in clinical trials (21). In the RIMA (Registre des infarctus du Maine-Anjou) study of 1,461 MI patients, only 34.8\% patients achieved the target-dose of $\beta$-blockers at discharge, and after one year follow-up, still nearly $60 \%$ of patients had not achieved the target-dose (22). A study of 208 post-MI patients, 154 of whom were treated with a mean $\beta$-blocker dose of $34 \%$ of the target-dose, demonstrated a $60 \%$ reduction in all-cause mortality at a mean follow-up of 58.5 months (23). Patients who had an acute MI and were treated with a lower dose of $\beta$-blockers than used in clinical trials may show similar or better survival than those given higher doses. The multicenter study enrolled 7,057 consecutive patients with acute MI at 26 centers in the United States and Canada (24). Researchers found no increased survival in patients treated with $\beta$-blocker doses approximating those used in previous randomized clinical trials compared with lower doses. These findings suggested that $\beta$-blocker may have a similar therapeutic effect at a lower dosage in comparison to the target-dose.

A previous study demonstrated that heterogeneous cardiac nerve sprouting and sympathetic hyperinnervation plays an important role in arrhythmogenesis and sudden cardiac death in both human patients and animal models of MI (25). Multiple randomized control trials have proven that $\beta$-blocker therapy could significantly reduce the incidence of sudden cardiac death (SCD) after MI. Part of the reason is that $\beta$-blockers reduce the activity of sympathetic system so as to decrease the level of plasma catecholamine. In the present study, we investigated the effects of early intravenous low- and target-doses of metoprolol on ventricular electrophysiological properties by using an acute MI canine model. Although significant diffe rence was identified between the low- and target-dose groups, similar tendency was observed in the therapeutic effect of both groups, indicating that the low-dose of metoprolol exhibited a similar effect on the ERP as well as the level of $\mathrm{NE}$ and $\mathrm{E}$ in animals with MI. These results suggested that low-dose $\beta$-blockers may exert a similar protective effect against ventricular arrhythmias after MI compared with the target-dose $\beta$-blockers. It indicated that low-dose practice maybe more reasonable in the $\beta$-blocker therapy against ventricular arrhythmias after MI due to lower toxicity and more safety. However, these data support the need for further testing. A further study will be conducted to determine optimal dosing of $\beta$-blockers after MI.

In conclusion, our study demonstrated that low-dose $\beta$-blockers exhibit similar effects to the target-dose on the improvement of ventricular electrophysiological properties through shortened ERP and decreased NE and E levels after MI. Thus, low-dose of metoprolol in $\beta$-blocker therapy may be reasonable due to similar effect and lower toxicity.

\section{Acknowledgements}

Not applicable.

\section{Funding}

This study was funded by the National Natural Science Foundation of China (General Program, 81270244; Beijing, China).

\section{Availability of data and materials}

The datasets used and/or analyzed during the present study are available from the corresponding author on reasonable request.

\section{Authors' contributions}

DNW, LW and DNL designed the study. DNW, LW and YH were responsible for the animal experiments and tests. $\mathrm{LH}$, 
$\mathrm{HMC}$ and PFC were responsible for the electrophysiological experiments. DNW, XL and JYZ were responsible for statistic analysis. DNW, LW and DNL wrote and finalized the study. All authors have read and approved the final manuscript.

\section{Ethics approval and consent to participate}

The study was approved by the Ethics Committee of Shanghai Changzheng Hospital (Shanghai, China).

\section{Patient consent for publication}

Not applicable.

\section{Competing interests}

The authors declare that they have no competing interests.

\section{References}

1. Go AS, Mozaffarian D, Roger VL, Benjamin EJ, Berry JD, Blaha MJ, Dai S, Ford ES, Fox CS, Franco S, et al; American Heart Association Statistics Committee and Stroke Statistics Subcommittee: Heart disease and stroke statistics - 2014 update: A report from the American Heart Association. Circulation 129: 399-410, 2014

2. Dawber TR, Moore FE and Mann GV: Coronary heart disease in the Framingham study. Am J Public Health Nations Health 47: 4-24, 1957.

3. Sivapalaratnam S, Boekholdt SM, Trip MD, Sandhu MS, Luben R, Kastelein JJ, Wareham NJ and Khaw KT: Family history of premature coronary heart disease and risk prediction in the EPIC-Norfolk prospective population study. Heart 96: 1985-1989, 2010.

4. Bolland MJ, Avenell A, Baron JA, Grey A, MacLennan GS, Gamble GD and Reid IR: Effect of calcium supplements on risk of myocardial infarction and cardiovascular events: Metaanalysis. BMJ 341: c3691, 2010.

5. Canto JG, Kiefe CI, Rogers WJ, Peterson ED, Frederick PD, French WJ, Gibson CM, Pollack CV Jr, Ornato JP, Zalenski RJ, et al; NRMI Investigators: Number of coronary heart disease risk factors and mortality in patients with first myocardial infarction. JAMA 306: 2120-2127, 2011.

6. Horne BD, Camp NJ, Muhlestein JB and Cannon-Albright LA: Identification of excess clustering of coronary heart diseases among extended pedigrees in a genealogical population database. Am Heart J 152: 305-311, 2006.

7. Hartiala J, Schwartzman WS, Gabbay J, Ghazalpour A, Bennett BJ and Allayee $\mathrm{H}$ : The genetic architecture of coronary artery disease: Current knowledge and future opportunities. Curr Atheroscler Rep 19: 6, 2017.

8. Assmann G, Cullen P and Schulte H: Simple scoring scheme for calculating the risk of acute coronary events based on the 10-year follow-up of the prospective cardiovascular Münster (PROCAM) study. Circulation 105: 310-315, 2002.

9. Gyárfás I, Keltai M and Salim Y: Effect of potentially modifiable risk factors associated with myocardial infarction in 52 countries in a case-control study based on the INTERHEART study. Orv Hetil 147: 675-686, 2006 (In Hungarian).

10. Basat O, Ucak S, Seber S, Oztekin E and Altuntas Y: After myocardial infarction carvedilol improves insulin resistance compared to metoprolol. Clin Res Cardiol 95: 99-104, 2006.

11. Tölg R, Witt M, Schwarz B, Kurz T, Kurowski V, Hartmann F, Geist V and Richardt G: Comparison of carvedilol and metoprolol in patients with acute myocardial infarction undergoing primary coronary intervention - the PASSAT Study. Clin Res Cardiol 95: 31-41, 2006.
12. Deljanin Ilic M, Pavlovic RF, Kocic G, Simonovic D and Lazarevic G: Effects of music therapy on endothelial function in patients with coronary artery disease participating in aerobic exercise therapy. Altern Ther Health Med 23: at5491, 2017.

13. Cai X, Bao L, Ding Y, Dai X, Zhang Z and Li Y: Quercetin alleviates cell apoptosis and inflammation via the ER stress pathway in vascular endothelial cells cultured in high concentrations of glucosamine. Mol Med Rep 15: 825-832, 2017.

14. Bischoff B, Silber S, Richartz BM, Pieper L, Klotsche J and Wittchen HU; DETECT Study-Group: Inadequate medical treatment of patients with coronary artery disease by primary care physicians in Germany. Clin Res Cardiol 95: 405-412, 2006.

15. Koch KC, Schaefer WM, Liehn EA, Rammos C, Mueller D, Schroeder J, Dimassi T, Stopinski T and Weber C: Effect of catheter-based transendocardial delivery of stromal cell-derived factor 1alpha on left ventricular function and perfusion in a porcine model of myocardial infarction. Basic Res Cardiol 101: 69-77, 2006.

16. Looi KL, Chow KL, Looi JL, Lee M, Halliday S, White H and Ellis C: Under-use of secondary prevention medication in acute coronary syndrome patients treated with in-hospital coronary artery bypass graft surgery. N Z Med J 124: 18-27, 2011.

17. Costalunga A and Gavazzi A: Effect of carvedilol on outcome after myocardial infarction in patients with left ventricular dysfunction: The CAPRICORN randomized trial. Ital Heart J Suppl 2: 1246-1247, 2001 (In Italian).

18. Gunnar RM, Bourdillon PD, Dixon DW, Fuster V, Karp RB, Kennedy JW, Klocke FJ, Passamani ER, Pitt B, Rapaport E, et al: ACC/AHA guidelines for the early management of patients with acute myocardial infarction. A report of the American College of Cardiology/American Heart Association Task Force on Assessment of Diagnostic and Therapeutic Cardiovascular Procedures (subcommittee to develop guidelines for the early management of patients with acute myocardial infarction). Circulation 82: 664-707, 1990.

19. Gislason GH, Rasmussen JN, Abildstrøm SZ, Gadsbøll N, Buch P Friberg J, Rasmussen S, Køber L, Stender S, Madsen M, et al: Long-term compliance with beta-blockers, angiotensinconverting enzyme inhibitors, and statins after acute myocardial infarction. Eur Heart J 27: 1153-1158, 2006.

20. Rochon PA and Gurwitz JH: Prescribing for seniors: Neither too much nor too little. JAMA 282: 113-115, 1999.

21. Ruta J, Ptaszyński P, Maciejewski M, Goch JH and Chizyński K: Effect of low doses of metoprolol, bisoprolol and carvedilol on mortality in patients with left ventricular dysfunction after acute myocardial infarction. Wiad Lek 59: 649-653, 2006 (In Polish).

22. Grall S, Biere L, Le Nezet M, Bouvier JM, Lucas-Chauvelon P, Richard C, Abi-Khalil W, Delepine S, Prunier F and Furber A: Relationship between beta-blocker and angiotensin-converting enzyme inhibitor dose and clinical outcome following acute myocardial infarction. Circ J 79: 632-640, 2015.

23. Siu CW, Pong V, Jim MH, Yue WS, Ho HH, Li SW, Lau CP and Tse HF: Beta-blocker in post-myocardial infarct survivors with preserved left ventricular systolic function. Pacing Clin Electrophysiol 33: 675-680, 2010.

24. Goldberger JJ, Bonow RO, Cuffe M, Liu L, Rosenberg Y, Shah PK, Smith SC Jr and Subačius H; OBTAIN Investigators: Effect of beta-blocker dose on survival after acute myocardial infarction. J Am Coll Cardiol 66: 1431-1441, 2015.

25. Miyauchi Y, Zhou S, Okuyama Y, Miyauchi M, Hayashi H, Hamabe A, Fishbein MC, Mandel WJ, Chen LS, Chen PS, et al: Altered atrial electrical restitution and heterogeneous sympathetic hyperinnervation in hearts with chronic left ventricular myocardial infarction: Implications for atrial fibrillation. Circulation 108: 360-366, 2003.

This work is licensed under a Creative Commons Attribution-NonCommercial-NoDerivatives 4.0 International (CC BY-NC-ND 4.0) License. 